\title{
Les Ursulines de Québec en 1850
}

\section{Marie-Emmanuel Chabot}

Volume 36, 1969

URI : https://id.erudit.org/iderudit/1007297ar

DOI : https://doi.org/10.7202/1007297ar

Aller au sommaire du numéro

Éditeur(s)

Les Éditions Historia Ecclesiæ Catholicæ Canadensis Inc.

ISSN

0318-6172 (imprimé)

1927-7067 (numérique)

Découvrir la revue

Citer cet article

Chabot, M.-E. (1969). Les Ursulines de Québec en 1850. Sessions d'étude - Société canadienne d'histoire de l'Église catholique, 36, 75-92.

https://doi.org/10.7202/1007297ar

Tous droits réservés @ Les Éditions Historia Ecclesiæ Catholicæ Canadensis Inc., 1970
Ce document est protégé par la loi sur le droit d'auteur. L'utilisation des services d'Érudit (y compris la reproduction) est assujettie à sa politique d'utilisation que vous pouvez consulter en ligne.

https://apropos.erudit.org/fr/usagers/politique-dutilisation/ 


\section{Les Ursulines de Québec en 1850}

Pour retracer l'histoire des Ursulines de Québec en 1850, il existe deux publications majeures : le quatrième volume de Les Ursulines de Québec depuis leur établissement jusqu'à nos jours, édité en 1866, et les Glimpses of the Monastery, publiés en 1897. Ces sources demeurent forcément incomplètes et du domaine de la chronique parce que les auteurs manquaient du recul nécessaire à toute œuvre exhaustive. Mais il nous reste les Annales de la Maison, et des liasses de lettres et de renseignements inédits qui feront les délices des historiens à venir. Plongée depuis quelques semaines dans ces précieux papiers, j'en ai presque oublié le manger et le dormir. Vous ne sauriez imaginer le bonheur qu'on éprouve à feuilleter les Archives après des années consacrées à l'enseignement et à la direction des études, tâches accaparantes, voire envoûtantes, mais incompatibles avec la recherche.

Après un survol du $\mathrm{XIX}^{\bullet}$ siècle, je constate que 1850 représente un sommet dans notre histoire, sommet qui suppose un mouvement ascensionnel. Il faut donc, pour comprendre cette ère de progrès, remonter, non pas à 1639 , ce qui serait le déluge, mais vers 1800 , et suivre les étapes de cette prodigieuse évolution.

Au point de départ, nous rencontrerons des inquiétudes, un état de détresse apparemment irréductible. Puis, grâce à des Ursulines exceptionnelles et à de nombreux bienfaiteurs, l'aurore percera les ténèbres et grandira jusqu'au plein midi.

\section{I. - PÉRIODE D'INQUIÉTUDES}

Dans le monde, le XIX ${ }^{\bullet}$ siècle s'ouvre d'une façon grandiose. A Rome, Pie VII vient de monter sur le trône pontifical et s'apprête à signer le Concordat avec la France. Napoléon, premier Consul, façonne à son gré les royaumes et les empires. L'Angleterre se dresse comme la première puissance du globe et les États-Unis d'Amérique se promettent de conquérir le monopole du commerce et de l'industrie. N'oublions pas, toutefois, que pendant le premier quart de siècle, l'Europe connaît des guerres presque incessantes.

Au Canada, la situation politique paraît instable, car la Constitution de 1791 n'a pas réglé tous nos problèmes coloniaux. La Loi de 1801, créant l'institution royale pour l'organisation d'un système primaire, 
s'accommode mal avec nos convictions religieuses. A cette époque, Londres ne reconnaît pas Mgr Plessis comme évêque de Québec, mais comme "Surintendant de l'Église romaine ".

Soixante ans après la Conquête, le Monastère des Ursulines de Québec éprouve, tout comme aux heures pénibles de l'Ancien Régime, de sérieuses inquiétudes au sujet de sa survivance. Il souffre d'abord d'une grande disette de sujets capables de soutenir l'œuvre de l'ensei-

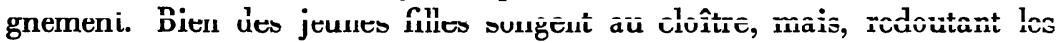
éventualités de la guerre, on leur refuse pendant neuf ans l'entrée du noviciat. En tout, la communauté ne comprend qu'une trentaine de religieuses professes. Se présente-t-il une postulante, il faut souvent tout lui donner, parce que sa famille ne peut payer de dot en argent ou en nature. Et l'on se plaint que les santés sont très faibles. Au fait, les Annales de cette période enregistrent la nécrologie de quelques Ursulines décédées dans la trentaine.

$D$ 'autre part, le nombre des élèves pensionnaires a sensiblement diminué. Elles arrivent vers l'âge de onze ou douze ans et restent juste le temps d'être instruites des choses de la religion.

Ruinées par la Guerre de Sept ans et la Révolution française, les Ursulines manquent de l'honnête nécessaire qui laisse pleine liberté de vaquer aux fonctions de l'enseignement. L'héritage d'Alençon, ancien patrimoine de Madame de la Peltrie, est définitivement perdu, et Monsieur Thavenet, chargé de constater la valeur relative des monnaies d'Angleterre, de France et du Canada nous a, par ses mauvais calculs, occasionné des pertes énormes. Souvent desservies par leurs Censitaires, les Ursulines décident, en 1801, d'affermer leurs Seigneuries de Portneuf et de Sainte-Croix. Les voilà délivrées d'une foule de peines et de dépenses qui excédaient leurs revenus.

Il arrive même que les différends aboutissent par-devant les tribunaux. En 1811, les Ursulines perdent un procès avec les Messieurs Allsopp qui se sont approprié depuis vingt-huit ans le passage d'une rivière de la Seigneurie de Portneuf rapportant un droit de péage à la communauté. En pareilles circonstances, il faut a bien travailler et bien ménager pour attraper les deux bouts ${ }^{1}$ ". Nos "Mères doreuses " sculptent et décorent des tabernacles, des statues et des chandeliers pour le Séminaire de Québec, pour les églises de la Province, notamment pour l'Islet et pour Saint-François de l'île d'Orléans qui conservent encore des pièces que nous avons pu admirer dernièrement. En 1809, on achète un "Christ neuf » pour le réfectoire au prix de douze louis

1 Les Ursulines de Québec depuis leur établissement jusqu'à nos jours, tome III, p. 83. 
que les jeunes religieuses paient avec leurs ouvrages en écorce. Ouvrages d'ailleurs très délicats et dignes d'être offerts à Lady Prévost (1815) ou à quelques visiteurs de marque. Pour refaire un escalier, on compte sur le revenu des classes qui consiste dans le louage des lits et des livres.

Dans ces conditions précaires, éclate la guerre de 1812. Les Ursulines partagent les anxiétés de leurs compatriotes :

Le Seigneur, soupire l'Annaliste, veut nous faire éprouver ou faire prendre une part plus sensible aux maux qu'ont essuyés et que subissent encore tant de pays ravagés par la guerre... Quelle tristesse et affligeante situation pour nous, pauvres religieuses, qui n'ayant aucune part aux différends et contestes des puissances de ce Monde, sommes sujettes néanmoins aux conséquences de leur domination ${ }^{2}$.

Mais quand la communauté change son horaire pour accommoder Monsieur French, aumônier d'un régiment irlandais, elle trouve une compensation dans la musique militaire :

Depuis le 16 octobre de cette année (1814), dit le Récit, nous n'avons tous les dimanches la messe qu'à sept heures, pour la commodité du régiment de la milice incorporée qui vient l'entendre dans notre église. Ils entrent au son des instruments, et continuent leur musique à diverses reprises pendant la Sainte Messe ${ }^{3}$.

Autre alerte le 16 octobre 1838 : Dodge et Theller, prisonniers d'État, tous deux condamnés à mort, s'évadent de la citadelle de Québec pendant la nuit. Un délateur prétend que ces Patriotes sont entrés chez l'avocat Morin, dans la rue du Parloir, et qu'ils ont sauté dans le jardin des Ursulines. Vite on court à l'évêché pour obtenir la permission de perquisitionner à l'intérieur du cloître. Des piquets de soldats entrent au couvent, fouillent partout, jusque dans les plus petites valises. Pendant ce temps, Dodge et Theller dorment dans une grange de la banlieue. Au Monastère et à la caserne, la folle équipée se termine par un éclat de rire.

\section{II. - DES SUJETS D’ÉLITE ET BIENFAITEURS}

Cet éclat de rire présage des jours meilleurs. Garder le sens de l'humour, c'est rester en excellente santé, disposer d'un potentiel assez fort pour secouer l'inertie et pour bondir vers le progrès. A condition, bien sûr, qu'un dynamisme extérieur vienne renforcer les réserves immanentes. D'ordinaire, le progrès comporte une évolution plus ou moins rapide, une croissance qui libère sans les froisser les puissances de la nature. Mais il y a des phénomènes uniques, des floraisons impatientes qui se moquent des saisons. Ainsi, les Ursulines de 1800 souhaitent un changement subit et radical, une espèce de revirement, voire un miracle de leur situation. La Providence les écoute en leur 
envoyant plusieurs sujets de langue anglaise déjà formés spécialement pour l'éducation de la jeunesse. Sur les cent soixante-quatorze professes du XIX ${ }^{\bullet}$ siècle, nous en comptons au moins cinquante. A la bonne heure, car plusieurs demoiselles anglaises, venues aux Ursulines pour apprendre le français, commencent à trouver les mêmes avantages dans les écoles protestantes, joints à celui d'étudier leur langue maternelle.

Notre premier sujet irlandais se nomme Elisabeth Dougherty. Née

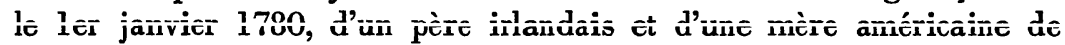
religion protestante, l'enfant fut baptisée à New York où M. Dougherty s'était établi pour éviter les sanctions pénales de son pays. Devenu veuf, il se chargea lui-même de l'éducation de sa fille et a s'appliqua à lui faire suivre les cours d'études propres aux collèges et aux académies. ${ }^{4}$ Après avoir éprouvé plusieurs revers de fortune, M. Dougherty décide de se fixer en Canada et obtint à Québec un emploi de traducteur.

Élisabeth a 15 ans lorsqu'elle entre pensionnaire chez les Ursulines pour étudier le français et se préparer à sa première communion. En trois ans, elle acquiert une connaissance " à peu près parfaite de la langue française et une rare habileté dans tous les genres de dessin et de broderie ${ }^{5}$ ». En 1798, elle opte pour le noviciat et se voit tout de suite entourée d'un groupe d'élèves avides d'apprendre la géographie, l'histoire et la langue anglaise. Remarquons que Mère Élisabeth Dougherty de Saint-Augustin est la première qui ait enseigné l'anglais aux Ursulines de Québec. C'est dire qu'il a fallu attendre trente-huit ans après la Conquête pour songer au bilinguisme. Mère Dougherty était une pédagogue si populaire que les élèves "désertaient en foule les écoles de la ville pour entendre ses leçons ${ }^{6}$ ". Malheureusement son zèle l'épuisa si tôt qu'elle mourut au printemps de 1814, dans la trentecinquième année de son âge.

Sa notice nécrologique la présente comme un "sujet précieux et une excellente religieuse qui aurait bien servi la religion, tant par ses vertus que par ses talents... L'Europe, où son père fut obligé de passer avec sa famille, contribua à lui faire acquérir beaucoup de connaissances. Elle avait l'esprit vif et pénétrant, de la mémoire et du goùt. Elle savait la grammaire anglaise et française, l'arithmétique, la géographie et réussissait très bien au dessin et à la peinture et à tout ce qu'elle entreprenait d'ouvrages à la main "."

Parmi les meilleures élèves de Mère Dougherty se trouvaient Mère Geneviève McKutcheon de Sainte-Hélène, dont la carrière se prolongea

Les Ursulines de Québec, tome IV, p. 621.

Les Ursulines de Québec, tome IV, p. 622.

Ibid.

7 Vieux Récit, p. 420. 
au-delà de cinquante ans, et Mère Marguerite Cuddy de Saint-Athanase, fille d'un officier irlandais en garnison à Québec. Pour entrer en religion, Marguerite dut rompre avec un jeune homme qui avait rêvé de l'épouser. En apprenant cette nouvelle, le fiancé lui écrivit :

Je n'oserais disputer au Roi des rois le cour si pur de sa jeune servante. Gardez, Mademoiselle, la foi que vous lui avez vouée et ne songez plus à votre premier engagement que pour recommander à Dieu votre serviteur ${ }^{8}$.

Une des figures les plus attachantes de cette époque est celle de Marie-Louise McLaughlin. Née à la Rivière-du-Loup en 1780, d'un père et d'une mère d'origine écossaise, elle était catholique et canadiennefrançaise de cour. Son grand-père, le colonel Simon Fraser, s'attacha à l'enfant et lui fit suivre les cours d'une école protestante. Enfin, l'aïeul céda aux instances des parents et consentit au départ de Marie-Louise pour le pensionnat des Ursulines. "Qu'elle essaie, dit-il, mais s'il lui prend la fantaisie de se faire religieuse, je déshériterai toute la famille! Fine mouche, la jeune fille profita d'une absence de son grandpère pour demander son entrée au noviciat. Elle avait pour garantie sa majorité, le consentement de ses parents et le bon cœur de Monsieur Fraser.

D'un esprit raffiné et d'une exceptionnelle énergie, Mère SaintHenri McLaughlin fit des efforts inimaginables pour hausser le niveau des études. Supérieure à quatre reprises, elle demeure une des gloires des Ursulines. Son frère, le Docteur David McLaughlin, résidant à Paris, la soutenait de ses conseils et de ses dons. C'est lui qui en 1836 fit don au Monastère de son premier orgue. Cette acquisition attira beaucoup de monde dans notre église. "La divine Providence qui a permis que nous eussions un orgue, remarque l'annaliste, nous a aussi pourvues de musiciennes dans la personne de nos chères Sœurs Marie-Jean et Marie-Benoît de Boston, et aussi de nos chères novices Sœur SainteCécile, professe, et Sœur Sainte-Philomène, voile blanc ${ }^{9}$."

Nous conservons un magnifique portrait à l'huile de Mère MarieLouise McLaughlin et des liasses de lettres que lui adressaient les abbés Desjardins, Thomas Maguire et Mgr Joseph-Octave Plessis. Les historiens qui ont l'habitude de considérer les revendications de Mgr Plessis ne soupçonnent peut-être pas la suavité de ses rapports avec les Ursulines. Après une visite canonique, il demande qu'on décharge les novices du soin du réfectoire pour leur procurer l'avantage de se " rendre capables des fonctions de notre institut ${ }^{10}$. Si les Ursulines lui fabriquent de l'encre et des ornements d'église, il leur rend ces

8 Vieux Récit, p. 446.

Annales, tome II, p. 193.

10 Annales, I, II, p. 36. 
délicatesses en dons substantiels, en visites-surprises. Par exemple, il décide de consacrer Mgr McDonell dans notre église.

Nous avons fini cette année 1820, dit le Récit, par la plus belle et la plus auguste cérémonie que nous eussions jamais osé espérer, et qui probablement ne se renouvellera plus dans notre église. Par une bienveillance toute particulière, Mgr de Québec a bien voulu y faire la cérémonie du sacre de Mgr Alex. McDonell, prêtre écossais qui, dès l'été dernier, avait reçu ses mulles du St-Siège avec le titre d'évêque de Résina, pour résider dans le Haut-Canada... Tout fut à la satisfactinn du nrélat ot dn clergé.

J'allais oublier de dire que Mgr nous donna pleine liberté d'avoir le voile levé pour mieux voir l'imposante cérémonie; nous profitâmes volontiers de cette permission 11 .

Parfois Mgr Plessis grondait ses filles de la rue du Parloir :

Pourquoi, écrivait-il un jour, divulguer tant d'avance qu'il doit y avoir une profession? Me voilà importuné de demandes pour l'entrée de votre monastère 12 .

Aux élections de 1821, une religieuse cumula deux charges et s'en excusa à la Supérieure qui souscrivit à sa demande. Trouvant la chose irrégulière, Mgr répondit :

Il faut respecter ce qui a été finalement arrêté entre les quatre premières élues et le supérieur majeur. Je ne vois nulle raison qui puisse autoriser à un autre arrangement. Vous voudrez bien signifier mon intention à qui il appartiendra. Quand les choses auront été mises sur le pied où elles étaient lorsque je vous quittai mercredi, j'approuverai tout le reste et vous irai voir. Ne m'attendez pas auparavant ${ }^{13}$.

Voilà pourquoi on ne pouvait jamais oublier que Mgr Plessis était évêque et supérieur du Monastère. Un des derniers billets que Mère Saint-Henri McLaughlin reçut de Mgr Plessis est en date de Sainte-Croix, 27 juin 1825 :

Ma Révérende Mère. Il n'est pas juste d'être dans la Seignerie de mes bonnes Ursulines sans leur donner signe de vie, surtout lorsque je sais tout l'intérêt qu'elles daignent prendre à ma santé. Vous saurez donc que je me porte bien, excepté le rhumatisme qui m'affecte les genoux et les pieds au point que je m'agenouille difficilement, et que je me relève plus difficilement encore. Bientôt je serai réduit à prier debout comme un Pharisien 14.

A ce moment, Mère Marie-Louise McLaughlin de Saint-Henri, supérieure, a 45 ans et Mère Cecilia 0'Conway de l'Incarnation, 37 ans. Celle-ci se trouve le dernière sur la liste des religieuses composant alors la Communauté. Son histoire tient presque de la légende. C'est le temps de dire que le Seigneur peut déplacer les montagnes pour exaucer les vœux de ses amis. Voyez plutôt.

11 Vieux Récit, p. 457.

12 Les Ursulines de Québec, I, IV, p. 578.

13 Ibid.

14 Les Ursulines de Québec, I, IV, p. 586. 
Cecilia 0'Conway naquit à Pittsburgh, en 1778, d'un père irlandais et d'une mère protestante qui fut déshéritée à cause de son mariage avec un catholique. Deux ans plus tard, elle entre dans le giron de l'Église. Après la confiscation de leurs biens, les O'Conway se réfugièrent en Amérique. En qualité d'interprète de langues, Monsieur O'Conway se rendit tour à tour à Baltimore, à Cuba, à Philadelphie, à la Nouvelle-Orléans. Cecilia accompagnait sa famille, apprit le français et l'espagnol en même temps que l'anglais. Sous la direction de son père, homme très érudit, elle étudia la religion, l'histoire, la géographie, la littérature et les sciences.

Mlle 0'Conway s'attacha d'abord à Madame Seton, fondatrice des Soeurs de la Charité de Baltimore. Cette dernière avait étudié la vie de Marie Guyart de l'Incarnation et en parlait volontiers à ses filles. En 1817, Sœur Cecilia fut envoyée avec quelques compagnes pour fonder la mission de New York. C'est là qu'elle rencontra nos religieuses en route pour la Nouvelle-Orléans. Dès 1822, elle rêve du Cloître des Ursulines de Québec. Mère Seton lui fait de douces remontrances, lui prouve que toute pensée de quitter la Congrégation se ramène à une pure tentation :

My own Cis, lui écrit-elle, all goes well and doubly well, for the cross fosters all within and without. Write me about yourself; may you enjoy the true peace in Him who has nailed us that your little poor Mother does !... He will take us home at last! Oh! my beloved Cis, then we will separate no more ${ }^{15}$.

Cette dernière phrase rappelle les paroles du cantique anglais :

Jerusalem, my happy home !

How do I sigh for thee!

Toujours attirée vers les Ursulines, Cecilia soupire :

Mon Dieu, si c'est votre volonté que j'aille à Québec, vous savez quels seront les transports de mon âme! Mais vous seul pouvez m'y conduire 16 .

Après la mort de Mère Seton, Cecilia se sent plus libre de changer de voie. Au début de 1823, elle demande son entrée au Monastère des Ursulines. Par prudence les moniales du temps en réfèrent à Mgr Plessis qui communique avec Mgr Connolly, évêque de New York. A la fin de février arrive la réponse suivante :

Quant à Cécile O'Conway, Sour de la Charité, dont V. G. me demande des informations dans votre lettre du 4 de ce mois (février), elle ne se trouve plus à New York, ayant été rappelée d'ici par ses Supérieures, pour aller vivre dans son couvent de St-Joseph dans le Maryland. Elle n'appartient donc plus à New York; mais je puis vous assurer qu'elle a été fort estimée ici de tous ceux qui ont eu l'occasion de la connaître, car elle est du naturel le plus heureux, fort pieuse, sage et bien instruite en tout ce qui concerne l'éducation 
de la jeunesse. De plus, elle est d'une humilité profonde et d'une douceur édifiante. Elle doit avoir environ 34 ans, et elle parle bien la ldngue française. Voilà $\mathrm{Mgr}$, ce que je puis vous dire avec sincérité à l'égard de Sour Cécile 17 .

On ne peut exiger de meilleur passeport. Comme bien l'on pense les Sœurs de la Charité s'opposent, craignent avec raison que ce précédent ne devienne une occasion de trouble pour la jeune Congrégation. Enfin les nuages se dissipent et Cecilia s'embarque pour le Canada. On comprend qu'elie puisse écrise.

My soul is almost overwhelmed with gratitude; I have no words to express what I feel : all that I can desire on this side of the grave is now presented to my hopes. I may now indeed in the joy of my soul exclaim with holy Simeon : Now, 0 Lord, dismiss thy servant in peace 18 ...

De leur côté, les Ursulines de Québec peuvent se réjouir du rare présent que la Providence leur envoie en la personne de Cecilia O'Conway. Ses longs voyages, ses vastes connaissances, son esprit d'observation joint à une mémoire étonnante et son habileté manuelle la rendent précieuse au pensionnat. A la cérémonie de profession, Mère Cecilia O'Conway de l'Incarnation prononce ses vœux d'Ursuline en présence de Mgr Plessis et de Madame la Comtesse Dalhousie. C'est la dernière fois que Mgr visite le monastère. Sœur Cecilia commence une seconde carrière qui durera près de quarante-deux ans.

Auprès des élèves, elle passait pour une encyclopédie vivante. Elle parlait d'abondance parce qu'elle avait connu plusieurs climats, collectionné des plantes et des pierres exotiques. Aux jours de congé, elle enseignait même l'espagnol aux privilégiées qui le désiraient. Avec ses élèves, elle assistait aux leçons de physique et de chimie de M. l'abbć Holmes, favorisant ainsi l'adoption d'un programme d'études plus vaste et plus riche.

Résumant la vie de cette grande Ursuline, l'Annaliste écrit :

Son ardeur pour le bien de cette maison, en même temps que pour sa propre perfection, ne s'est jamais lassée; et nous pouvons dire que si son entrée lui parut un miracle de la protection de Dieu sur elle, ce fut aussi pour notre institut une marque des soins de la divine Providence 19.

Pour continuer la galerie de nos portraits monastiques, je vous présente maintenant les Mères Mary et Abby Barber. Leur histoire familiale ressemble à celle des Van der Meer. Leur père, le Révérend Virgil-Horace Barber, pasteur épiscopalien, épousa Miss Jeruska Booth, femme d'une haute intelligence. Cinq enfants naquirent de ce mariage.

17 Les Ursulines de Québec, I, IV, p. 542.

18 Ibid., p. 543.

19 Les Ursulines de Québec, I, IV, p. 544. 
Un jour, le pasteur Barber se met à lire la vie de saint François-Xavier. Le voilà ému, intrigué : Qui donc a pu lui inspirer pareil héroïsme ? Commence alors une longue période de recherches qui se termine par la conversion au catholicisme de toute la famille. Bientôt, M. Barber se sent appelé à la prêtrise, part pour Rome où il entre au noviciat des Jésuites. Entraînée dans la même voie, Madame Barber prend le voile à la Visitation de Georgetown. Les trois aînées, Mary, Abby et Suzanne sont admises comme pensionnaires à ce couvent. Madame Jenwick, mère de trois Jésuites, prend soin des deux petits, Samuel, âgé de trois ans et Joséphine, bébé de dix mois.

Le 23 février 1820, une cérémonie extraordinaire réunissait un nombreux public dans la chapelle du couvent de la Visitation. Sur des sièges réservés, on voyait cinq enfants...; tous étaient graves et tristes; leur mère, Soeur Saint-Augustin (Mme Barber) s'avança vers la grille et prononça les trois voux de pauvreté, de chasteté et obéissance; immédiatement après, M. Barber lut à haute voix la formule de profession des fils de saint Ignace 20.

Cinq ans plus tard, Mary et Abby Barber font leur choix de vie : elles seront ursulines, l'une à Boston et l'autre à Québec. Suzanne deviendra ursuline de Trois-Rivières, Joséphine, visitandine et Samuel, jésuite.

En 1834, un incendie allumé par des mains criminelles rase le Couvent des Ursulines de Boston. Aussitôt le Monastère de Québec ouvre ses portes aux religieuses américaines. Mère Mary Benedict Barber se trouve pour toujours sous le même toit que sa sœur Abby. Mère Benedict vient grossir le nombre des professeurs qualifiés qui œuvrent dans notre institut entre 1835 et 1847.

Elle enseigne la littérature anglaise et la diction. C'est elle qui propage l'écriture anguleuse et légèrement penchée que plusieurs générations de nos élèves adopteront.

Non moins douée, Mère Abby Barber se dépense auprès des jeunes élèves. Artiste, elle a laissé des broderies d'une rare perfection. A cause de son originalité et de sa valeur humaine, la correspondance de la famille Barber mérite d'être éditée. A titre d'exemple, citons la finale d'une lettre que le Père Barber adressait à sa fille Suzanne, le 31 décembre 1836 :

Avec la même tendresse avec laquelle, pendant tant d'années, je vous ai portée dans mes bras et pressée sur mon cour, je suis toujours, ma très-chère Suzanne, votre affectueux père. 21

La plupart des renseignements au sujet des Ursulines anglaises de la première partie du $\mathrm{XIX}^{\bullet}$ siècle nous viennent de Mère Sainte-Croix

20 Les Ursulines de Trois-Rivières, tome II, p. 305-306.

21 Les Ursulines de Trois-Rivières, tome II, p. 326. 
Holmes, auteur des Glimpses of the Monastery. Au début des pages qu'elle consacre en appendice aux années 1839 à 1889, elle délaisse la tradition et les Annales pour s'appuyer sur sa propre expérience, sur ce qu'elle a vu et touché : «We can now tell of what we have seen and testify to what we have known ${ }^{22}$."

Sœur du célèbre abbé John Holmes, connu comme orateur sacré et promoteur de l'Université Laval, Marie-Suzanne-Joséphine Holmes se

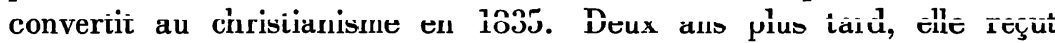
l'habit des Ursulines et prit le nom de Sainte-Croix. Nos doyennes de 1969 qui l'ont connue vers 1910, dans sa 94e année, nous disent qu'elle était d'une stature impressionnante, d'une intelligence aussi remarquable pour les lettres que pour les sciences. Elle apprit quatre langues et donnait des leçons dignes du niveau collégial. En 1840, elle dessina un planisphère de 5 par $41 \frac{1}{2}$ pieds; reproduit en 1896, il fut primé à l'exposition universelle de Chicago.

Mère Sainte-Croix Holmes devait une large part de ses succès pédagogiques à son frère, M. l'abbé Holmes (1799-1852), l'un des prêtres les plus éminents du Séminaire de Québec. Agrégé à 28 ans, il enseignait avec la même facilité l'hébreu, le grec, le latin et les sciences naturelles. La musique, le dessin, l'art oratoire, l'art dramatique, tout l'intéressait. Il clamait contre l'arriérisme des programmes. En un tournemain il mit son collège à la page. Partout on l'aimait, même s'il paraissait bousculer certaines traditions et devancer son temps.

Outre les immenses services qu'il rendit à sa propre institution et à l'Église canadienne tout entière, M. Holmes prit à cœur les intérêts des Ursulines. Il s'occupa du règlement des difficultés concernant nos domaines illégalement expropriés lors de la Révolution française. Ses commandes de livres et l'instruments se faisaient souvent en double. Volontiers il nous passait ses manuscrits, son Histoire moderne, son Histoire ancienne, son Abrégé de Géographie moderne dont la plupart des collèges ont fait usage jusqu'au-delà de 1900. Pour relever l'enseignement primaire, déchu après la Conquête, il mit sur pied deux écoles normales (1836), l'une aux Ursulines, l'autre à Montréal.

Au milieu de ses accablantes besognes, M. Holmes trouvait des loisirs pour enseigner au parloir des Ursulines. Quand Mgr Signay, enchanté du succès des élèves, nous fit don d'une machine pneumatique, " M. Holmes eut la bonté d'en donner des leçons ${ }^{23}$ ". Entre 1833 et 1848 , il nous confia cinq de ses seurs qui reçurent le baptême dans notre chapelle.

Glimpses of the Monastery, Reminiscences of fifty years in the Cloister, p. 1. Annales, I, II, p. 166. 
Son grand ami, M. Horan, fut aussi un bienfaiteur de notre maison. En 1848, il dirige les ouvriers qui posent le gaz pour la première fois dans notre chapelle. Pendant son stage à l'Université Yale et dans ses randonnées subséquentes, il n'oublie pas les Ursulines. Nous lui devons plusieurs feuillets de nos herbiers et de nombreux spécimens de nos collections de géologie. Devenu évêque de Kingston, il continue de cultiver ses amies de la rue du Parloir.

Les religieuses anglaises enrichirent notre bibliothèque d'une foule de volumes que nous conservons aux Archives. Entre autres, mentionnons les collections Pennock et Irwing.

D'année en année le pensionnat acquiert de l'importance à cause des différentes branches qui s'y enseignent par principes et d'une manière solide. On réussit à mettre les classes " sur le pied de la règle, c'est-à-dire par gradation ${ }^{24}$ ». Le nombre des élèves monte en flèche : en 1822, on compte 80 pensionnaires et 250 externes. En 1836, on reçoit 110 pensionnaires. Un coup d'œil sur le Prospectus de 1847 nous aide à reconstruire la physionomie du pensionnat. L'uniforme des dimanches et des fêtes est une robe de mallemole pendant l'été et une robe de tartan pendant l'hiver. Aux autres jours, la règle prescrit, tant pour l'hiver que pour l'été, une robe de tartan. Quant aux cheveux, ils se portent courts ou longs suivant le goût des parents, mais l'usage des papillottes est strictement défendu. Même interdiction pour le thé, " poison mortel pour les personnes du sexe qui en font un usage habituel, surtout lorsqu'elles le prennent à jeun le matin ${ }^{25}$ ». On se demande si ce point du règlement n'a pas été inspiré par le docteur Farges, ancien médecin du Monastère, qui refusait « de donner ses soins à celles de ses malades qui conservaient l'usage du thé ${ }^{26}$ ". Pour obéir, les Ursulines obtinrent de Mgr de Sidyme la permission de boire du chocolat au déjeuner.

En 1847, le cours d'instruction renferme :

La lecture, l'écriture, l'arithmétique, la tenue des livres, la grammaire française, la grammaire anglaise, la rhétorique, la composition française, la versification anglaise, la versification française, l'histoire ancienne, l'histoire moderne, l'histoire sacrée, la mythologie, la cosmographie, la géographie, l'usage des globes et des cartes géographiques, les éléments d'astronomie, de physique, de botanique et de chimie, l'orgue, la harpe, le piano, la guitare et l'accordéon, la musique vocale, le dessin, la peinture à l'huile, à l'aquarelle, à la gouache, en miniature, en pastel et en crayon, la peinture sur le satin, ouvrages à l'aiguille et broderie de tout genre, fleurs et fruits artificiels etc. L'éco- 
nomie domestique dans ses différentes branches est un objet auquel on exerce les élèves avec soin.

Prix : $\$ 66.00$ pour le pensionnat

$\$ 33.00$ pour le demi-pensionnat 27 .

Il va sans dire que ce riche prospectus implique de la part des religieuses une solide culture. Pour se perfectionner dans l'aquarelle, le dessin et la peinture, les artistes du cloître se mirent à l'école du colonel Colborne. de M. Bowman, peintre bostonnais envoyé par Mgr Fenwick.

A la demande de Mgr Signay, les Ursulines s'adonnèrent même à l'architecture, art que Sa grandeur trouvait " aussi utile au sexe féminin qu'au masculin ${ }^{28}$ ". A partir de ce moment, on cultive l'enluminure.

Comme professeurs de musique, les Ursulines eurent M. Frederick Glackmeyer, maître de bande d'un des régiments des troupes auxiliaires de Brunswick, M. Codman, organiste de la cathédrale anglicane et Mme 0'Madden, Sœur de Mère Cecilia 0'Conway.

Cependant, c'est surtout à M. Jean-Denis Daulé qu'on doit l'inauguration des cantiques à la chapelle. Né à Paris en 1776, M. Daulé était entré à la Trappe de Sept-Fonts qui n'avait jamais rencontré de novice aussi enjoué. Après un accès de fou rire, il reçut son congé et reprit sa théologie comme prêtre séculier. Au Canada, il travailla comme missionnaire puis comme curé des Écureuils jusqu'à son installation comme aumônier du Monastère en 1806. Devenu presque aveugle, il obtint sa démission en 1832.

Il quitta notre Maison le 14 mai, à son grand regret et au nôtre, écrivait Mère St-Henri McLaughlin. Vingt-six ans de résidence avaient formé entre le saint prêtre et notre communauté des liens de charité qui firent couler bien des larmes... Il a beaucoup travaillé la nuit aux dépens de sa vue pour rédiger un recueil de cantiques, avec notes, pour le diocèse, ouvrage bien adapté aux besoins, et dont plusieurs morceaux sont de sa composition 29 .

Si les Ursulines et leurs élèves profitaient des leçons de M. Daulé, M. l'abbé de Calome, aumônier des Ursulines de Trois-Rivières, le sermonait vertement :

Mon ami, lui écrivait-il, je ne suis pas ennemi des délassements, ils sont nécessaires; mais je n'aime pas votre violon. Je vous l'ai déjà dit, son moindre mal c'est d'exposer à la perte du temps, et le vôtre est très précieux. Vous me parlerez du Roi David et de Sainte Cécile. Un roi ! une femme! à la bonne heure. Mais vous, prédicateur et directeur... comment au milieu de tant de devoirs et de bonnes œuvres trouvez-vous du temps pour jouer le violon ${ }^{30}$.

27 Prospectus de 1847.

28 Annales, tome II, p. 134.

29 Annales, II, p. 95.

30 Les Ursulines de Québec, tome IV, p. 456. 
Et l'annaliste d'ajouter avec un sourire: "Ce violon tant persécuté faisait pourtant grand honneur à nos fêtes monastiques ${ }^{31}$.

D'une façon différente mais non moins importante, les deux messieurs Desjardins, réfugiés au Canada après la Révolution, rendirent d'importants services aux Ursulines. L'aîné, M. Philippe-Jean-Louis Desjardins fut grand vicaire de Mgr Hubert, chapelain des religieuses de l'Hôtel-Dieu et directeur des Ursulines. En 1802, les médecins déclarèrent que notre climat lui était contraire et prescrivirent comme seul remède efficace un prompt retour en France. "Aveu tacite de leur peu d'habileté », souligne l'annaliste mécontente du verdict de la Faculté. M. Philippe-Jean-Louis Desjardins ne revint plus au pays. Longtemps après son départ, il entretint avec ses amies du Monastère une correspondance débordante d'amitié et de savoureux détails historiques. Ainsi, il nous apprend que les Ursulines préparaient d'excellentes gelées, qu'elles importaient du fer-blanc pour couvrir les toits, qu'Antoine Plamondon commençait à devenir célèbre. De 1810 à 1814, brusque interruption de la correspondance: M. Desjardins payait de la prison et de l'exil l'amitié que lui avait vouée le duc de Kent. Napoléon ne tolérait pas pareil commerce de lettres entre un Français et un prince anglais.

En M. l'abbé Joseph Desjardins, frère cadet de Philippe, les artistes et les Ursulines trouvèrent un ami secourable et généreux. Supérieur de notre maison de 1825 à 1833 , il nous distribua des toiles de maîtres expédiées de France par son frère. Nous leur devons Le Repas du Sauveur chez le Pharisien et le Sauveur prêchant de Philippe de Champagne, la Parabole des dix Vierges, la Pêche Miraculeuse, tableaux qui se trouvent dans notre église. Comme bien l'on pense, ces tableaux coûtaient relativement cher en 1830 , mais on se les procurait à des conditions raisonnables. A preuve : pour Le Souper chez le Pharisien, on demande 100 louis payables en vingt ans. A propos de cette toile, M. Gérard Morisset écrit :

L'église des Dames Ursulines ne contiendrait-elle que la somptueuse pièce de Philippe de Champagne - cet inoubliable Repas chez Simon le Pharisien - qu'elle devrait attirer les amateurs de bonne peinture, même et surtout ceux qui ont eu la bonne fortune de visiter les plus beaux musées de l'Europe et de l'Amérique. Qui, au XVII siècle, se serait douté que ce chef-d'œuvre pourrait être transporté par le tourbillon de la Révolution jusqu'à l'autre bout du monde, dans un Monastère d'Ursulines ${ }^{32}$.

Le tome quatrième de l'Histoire du Monastère se clôt sur un chapitre consacré à M. l'abbé Thomas Maguire, "Second fondateur ".

31 Les Ursulines de Québec, tome IV, p. 456.

32 Gérard Morisset, Le Canada français, vol. XXII, $\mathrm{n}^{\circ}$ 9, mai 1933, La Collection Desjardins au Couvent des Ursulines. 
Aumônier des Ursulines pendant vingt-deux ans, M. Maguire mériterait qu'on lui consacre un volume tout entier. Né à Philadelphie le 9 mars 1776, d'un père catholique et d'une mère protestante, il étudia au Séminaire de Québec. Encore diacre, il fut nommé secrétaire de Mgr Denaut. Après son ordination en 1799 , il devint vicaire à la cathédrale, missionnaire à Tadoussac et à Chicoutimi, Grand Vicaire de Mgr Panet, curé de Saint-Michel, supérieur du Collège de Saint-Hyacinthe et aumônier des Ursulines (1825-1854). Au cours de ses voyages en Europe, il débrouilla les affaires des communautés religieuses, acheta des livres, des ornements, un orgue et une harpe pour les Ursulines.

A Paris, M. Maguire rencontra Mlle Moore, ancienne élève des Ursulines du Faubourg Saint-Jacques, qui, lors de la Révolution, s'était procuré plusieurs souvenirs de ses anciennes Maîtresses avec l'intention de les remettre. M. Maguire s'empressa d'acquérir le tout pour les Ursulines de Québec. Ces archives manuscrites comprennent les Annales des cinquante premières années des Ursulines de Paris (1612-1662), douze registres, plusieurs cahiers concernant le Gouvernement et l'administration du monastère et un grand nombre de volumes spirituels.

Après cette transaction, Mlle Moore écrivait au curé de Compiègne :

Je suis charmée de pouvoir vous dire combien je suis heureuse de voir les Ursulines de Québec propriétaires de ces restes précieux de l'ancienne maison de leurs Mères. Je ne désespère pas maintenant de la voir un jour relevée par elles $\mathbf{3 3}$.

Plus que tout autre, M. Maguire s'est dévoué pour le bien spirituel de notre communauté, le redressement de nos finances et l'épanouissement du pensionnat. Il examina les titres de nos propriétés, nos dettes, nos revenus, nos dépenses, et l'effet des spoliations. Pour enrayer la crise financière, il imposa seize mesures d'économie. Il fit construire, "dans un des plus beaux quartiers de la ville ${ }^{34}$ ", trois maisons qui se louaient cent louis par an. M. Maguire rédigea lui-même des règlements pour les élèves. C'est lui qui inaugura au Monastère la pratique des examens publics qui duraient trois jours. Le premier se déroula le 31 janvier 1837 dans la chambre de l'aumônier, en présence d'une vingtaine de prêtres. Mlle Joséphine Holmes remporta le prix d'excellence, tant pour ses progrès dans différentes branches que pour sa bonne conduite. Le tout se termina par la tragédie d'Esther.

Deux ans plus tard, on ajouta des expériences d'électricité, de la musique instrumentale et vocale et le drame de Coriolan. Trait bien féminin : on avait tendu un rideau au bas de la salle, afin de donner aux

33 Annales, tome II, p. 174.

34 Ibid., p. 240. 
maîtresses la facilité d'entendre les élèves et de se « dérober à la vue du public ${ }^{35}$ 》.

Pour célébrer le deuxième centenaire, on exécuta une Messe de Mozart. Et en 1848, on joua Polyeucte à la distribution des prix.

Formées pour vivre dans le monde, les filles des Ursulines avaient souvent l'occasion de recevoir des personnages de marque comme Lady Prevost, la comtesse de Dalhousie et Lady Aylmer. Celle-ci, dans ses Recollections rapporte qu'elle présida deux ou trois examens publics aux Ursulines. Elle décora même les élèves les plus méritantes de la Croix de Saint-Louis.

Puisqu'il s'agit de Lady Aylmer, notons que son époux fit enclaver (1831), dans le mur de notre église, du côté de l'épître, à la mémoire de Montcalm, l'écusson qui porte l'inscription suivante :

\author{
Honneur à Montcalm \\ Le destin en lui dérobant la victoire \\ l'a récompensé par \\ une mort glorieuse
}

En mai 1833, on ouvrit la tombe du général :

Tout s'y trouva dans un état de décomposition complète, à l'exception du crâne que l'on recueillit avec respect et qui se conserve dans le musée ${ }^{36}$.

Mais revenons à M. Maguire. Il nous a laissé un relevé de nos bâtiments de 1850. En suivant ses plans, on comprend bien l'évolution du Monastère depuis 1642. Le premier couvent construit par Marie de l'Incarnation brûle en 1650. Le second date de 165l. Un nouvel incendie endommagea l'édifice, mais les murs restèrent debout. En 1832, on éleva un troisième étage. L'aile Sainte-Famille commencée en 1686 fut achevée en 1688. Le prolongement de cette aile se fit entre 1712 et 1715. Notre première grande église date de 1723. En 1836, on rebâtit la Maison de Mme de la Peltrie et l'on construisit l'Aile Sainte-Angèle.

C'est à la suggestion de M. Maguire que les Ursulines firent appel à la Législature pour obtenir des subsides. Au lieu d'adresser une froide requête à ces Messieurs, on les invita aux examens publics. Le 14 janvier 1836, le Canadien publiait un compte rendu de la réception de la veille :

Hier l'après-midi, M. l'Orateur et presque tous les membres de la Chambre d'Assemblée présents en ville, furent visiter le couvent des dames Ursulines de cette ville. Les élèves de ces dames répétèrent devant ces messieurs une pièce dramatique sacrée, qui aurait seule, par la manière dont elle fut débitée, donné la plus haute idée de l'éducation classique que les jeunes personnes du sexe puisent dans cette pieuse institution. Les ouvrages de dessin et à l'aiguille, et dans tous les genres 
qui constituent l'éducation d'une demoiselle, que les honorables visiteurs eurent l'occasion d'examiner, portèrent l'admiration à son comble, et tous revinrent enchantés, et des élèves et de l'affabilité des Dames aux soins zélés et éclairés desquelles elles sont confiées.

Ce n'est pas d'aujourd'hui que le Bas-Canada, que Québec ont à se féliciter de posséder une des meilleures maisons d'éducation en tous genres pour les demoiselles, qui existent sur ce continent, et en Europe peut-être. Aussi, la Législature s'est-elle toujours empressée de seconder les efforts de ces Dames, toutes les fois qu'elles ont eu besoin de secours extraordinaire pour étendre le bienfait de l'œuvre méritoire à laquelle elles sont vouées ${ }^{37}$.

En octobre 1849, deux Ursulines de Québec partirent pour le nouveau couvent de Galveston. Comme il l'avait fait en 1822 pour les Ursulines en route pour la Nouvelle-Orléans, M. Maguire voulut bien les accompagner jusqu'à New York. Cédant aux instances des Missionnaires du Texas, il fit tirer son portrait au daguerréotype. Théophile Hamel se servit de cette miniature pour peindre les traits du vénérable octogénaire. Cette toile fort bien réussie orne encore les murs de notre salle de communauté.

Monsieur Thomas Maguire mourut le 18 juillet 1854, à l'âge de 80 ans. Il avait, paraît-il, refusé trois fois la mitre. Au cours de sa longue carrière, il connut cinq évêques canadiens : Mgr Denant qui le choisit comme secrétaire, Mgr Plessis qui le nomma vicaire à la cathédrale de Québec, Mgr Panet qui en fit son grand vicaire et Mgr Signay qui le députa en Europe pour obtenir la nomination de Mgr Turgeon. En terminant l'éloge de M. Maguire, l'Annaliste a bien raison d'écrire :

Sa mémoire, comme celle du juste, vivra aussi longtemps que subsistera ce Monastère; et si nous laissons à celles qui viendront après nous de publier le détail d'une vie dont tous les moments furent dévoués à la Religion et au pays, c'est que ces bienfaits sont encore tout payables, et que leur importance ne peut que se développer avec le temps 38 .

Tout compte fait, ce rapport des cinquante premières années du XIX $^{e}$ siècle peut laisser l'impression que le Monastère des Ursulines doit toute sa prospérité aux sujets venus d'Irlande, des Etats-Unis et du Haut-Canada. Pourtant, que les Séparatistes se consolent, le Québec est fort bien représenté dans notre histoire monastique de 1800 à 1850. Nous lui devons des femmes remarquables comme la Mère Marie-AnneLouise Taschereau de Saint-François-Xavier, « issue de parents les mieux qualifiés de cette ville ${ }^{39}$ "; les Mères Ursule Marchand, Geneviève et Marie-Thérèse Berthelot, François Panet, Adélaïde Plante, supérieure vingt-quatre ans, Émilie Miville-Dechêne, Adèle Cimon, annaliste et auteur de l'Histoire du Monastère. Et n'oublions pas la pléiade des religieuse canadiennes-françaises, élèves de ces grandes Ursulines.

37 Le Canadien, 14 janvier 1836.

38 Les Ursulines de Québec, tome IV, p. 728.

39 Annales, tome II, p. 37. 
Parmi les femmes du Monde, saluons les épouses de PhilippeAubert de Gaspé, de Louis-Joseph Papineau, de Sir Étienne Taché, de l'honorable juge Elzéar Bédard, de Sir Louis-Hippolyte La Fontaine, de Sir Thomas Chapais.

Dans la première partie du XIX ${ }^{\bullet}$ siècle, les Ursulines de Québec n'acceptèrent aucune fondation, mais elles envoyèrent des sujets à la Nouvelle-Orléans (1822), répondant à l'appel de Mgr Dubourg qui avait demandé "des religieuses de 38 à 43 ans, exemptes de rhumatismes, de maux de tête, de délicatesse de nerfs ${ }^{40}$ \%.

En 1824, la Mère Ursule Moffet de Saint-Georges sortit du Monastère pour aller gouverner celui de Boston. Et deux Ursulines de Québec partirent pour le Texas en 1849. C'est dire que nous avons tenté l'impossible pour témoigner notre gratitude aux Américains. Ces échanges de sujets présageaient les grandes unions du $\mathrm{XX}^{\bullet}$ siècle entre les monastères d'Ursulines du Québec et du monde entier.

Le 19 août 1850, la foudre déchiqueta le vieux frêne sous lequel Marie de l'Incarnation catéchisait les Indiennes :

La terre fut longtemps jonchée de débris, chacune pouvant à loisir recueillir des reliques de cet ami du passé.

Il nous reste aussi à recueillir les trésors des trois derniers siècles. Nos archives s'organisent d'une façon moderne et toute scientifique. Souhaitons qu'une équipe de spécialistes entreprenne bientôt une nouvelle édition de l'Histoire des Ursulines de Québec.

En attendant, je tiens à vous présenter quelques personnages du $\mathrm{XIX}^{\bullet}$ siècle, invités d'honneur au Congrès de la Société canadienne d'Histoire de l'Église.

Ce sont :

- Monsieur l'abbé Philippe Desjardins

- Monsieur l'abbé Thomas Maguire

- Mgr Joseph-Octave Plessis

- Lord Aylmer

- Lady Aylmer

- Lady Prevost

- Mère Marie-Louise McLaughlin

Marie-Emmanuel Chaвot, o.s.u.

Docteur en lettres et en philosophie.

40 Ibid., p. 2. 


\section{BIBLIOGRAPHIE}

American Catholic Historical Society, vol. X, tome III.

Archives Manuscrits des Ursulines de Québec de 1800 à 1850.

Mère S. Joseph Barnard, o.s.u., Un pèlerinage des Ursulines de Québec dans le domaine de saint Joseph. Cahiers de Joséphologie, vol. XIII (1965) ; vol. XVI (1968). Centre de Recherche et de Documentation, Oratoire Saint-Joseph, Montréal.

Morisset, Gérard, La Collection Desjardins au couvent des Ursulines, Le Canada français, vol. XXII, $\mathrm{n}^{\circ}$ 9, mai 1933, p. 855-868.

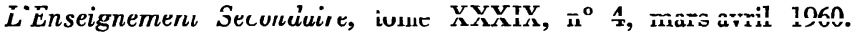

Glimpses of the Monastery, Scenes from the history of the Ursulines of Quebec during two hundred years (1639-1839). Second edition revived, augmented and completed by Reminiscences of the last fifty years (1839-1889), Québec, Demers, 1897.

WaDE, Mason, The Contribution of Abbe Holmes to Education in the Province of Quebec. Culture, XV, 1954.

Roy, Pierre-Georges, A travers l'histoire des Ursulines, Lévis, 1939.

Roy, Pierre-Georges, Bulletin des Recherches historiques, vol. III, Lévis, 1897; vol. XXII, $\mathbf{n}^{\circ}$ VII, 1916.

Mgr TÊtu, Les évêques de Québec, Québec, Hardy, 1889.

Trudel, Marcel, L'Eglise canadienne, t. II.

Les Ursulines de Québec depuis leur établissement jusqu'à nos jours, tome III et IV, Québec, Darveau, 1866.

Les Ursulines des Trois-Rivières, t. II. Trois-Rivières, Ayocte, 1892. 